\title{
A GENERATING FUNCTION OF THE SQUARES OF LEGENDRE POLYNOMIALS
}

\author{
WADIM ZUDILIN
}

(Received 28 November 2012; accepted 6 February 2013; first published online 22 March 2013)

\begin{abstract}
We relate a one-parametric generating function for the squares of Legendre polynomials to an arithmetic hypergeometric series whose parametrisation by a level 7 modular function was recently given by Cooper. By using this modular parametrisation we resolve a subfamily of identities involving $1 / \pi$ which was experimentally observed by Sun.
\end{abstract}

2010 Mathematics subject classification: primary 33C20; secondary 11F03, 11F11, 11Y60, $33 \mathrm{C} 45$. Keywords and phrases: $\pi$, Legendre polynomial, generating series, binomial sum, modular function.

\section{Introduction}

In our joint papers [7] with Chan and Wan and [14] with Wan we made use of, but also extended, the generating functions of Legendre polynomials

$$
P_{n}(y)={ }_{2} F_{1}\left(\begin{array}{c|c}
-n, n+1 & \frac{1-y}{2} \\
1
\end{array}\right),
$$

originally due to Brafman [3]. Our generalised generating functions have the form $\sum_{n=0}^{\infty} u_{n} P_{n}(y) z^{n}$, where $u_{n}$ is a so-called Apéry-like sequence, as well as

$$
\sum_{n=0}^{\infty}\left(\begin{array}{c}
2 n \\
n
\end{array}\right)^{2} P_{2 n}(y) z^{n} \quad \text { and } \quad \sum_{n=0}^{\infty} \frac{(3 n) !}{n !^{3}} P_{3 n}(y) z^{n} .
$$

One motivation for the work was a list of formulas for $1 / \pi$ given by Sun [13]. Because the preprint [13] is a dynamic survey of continuous experimental discoveries by its author, a few newer examples for $1 / \pi$ involving the Legendre polynomials appeared after acceptance of [7, 14].

Namely, two groups of identities related to the generating functions

$$
\sum_{n=0}^{\infty} P_{n}(y)^{3} z^{n} \quad \text { and } \quad \sum_{n=0}^{\infty}\left(\begin{array}{c}
2 n \\
n
\end{array}\right) P_{n}(y)^{2} z^{n}
$$

The author was supported by the Australian Research Council.

(c) 2013 Australian Mathematical Publishing Association Inc. 0004-9727/2013 \$16.00 
are now given as (VI1)-(VI3) and (VII1)-(VII7) on p. 23 of [13]. A search of the existing literature on the subject reveals no formula which could be useful in proving Sun's observations. The closest possibility is Bailey's

$$
\begin{aligned}
\sum_{n=0}^{\infty} P_{n}(x) P_{n}(y) z^{n}= & \frac{1}{\left(1+z\left(z-2 \sqrt{\left(1-x^{2}\right)\left(1-y^{2}\right)}-2 x y\right)\right)^{1 / 2}} \\
& \quad{ }_{2} F_{1}\left(\frac{1}{2}, \frac{1}{2} \mid \frac{-4 \sqrt{\left(1-x^{2}\right)\left(1-y^{2}\right)} z}{1+z\left(z-2 \sqrt{\left(1-x^{2}\right)\left(1-y^{2}\right)}-2 x y\right)}\right),
\end{aligned}
$$

which follows from [2, Eqs. (2.1) and (3.1)] and [1, Eq. (7) p. 81]. The generating function (1.2) was rediscovered later by Maximon [10]. It admits, in fact, a less radical form

$$
\sum_{n=0}^{\infty} P_{n}(x) P_{n}(y) z^{n}=\frac{1}{\left(1-2 x y z+z^{2}\right)^{1 / 2}}{ }_{2} F_{1}\left(\frac{1}{4}, \frac{3}{4} \mid \frac{4\left(1-x^{2}\right)\left(1-y^{2}\right) z^{2}}{\left(1-2 x y z+z^{2}\right)^{2}}\right)
$$

which is due to Wan (private communication, 27 September 2012). Unfortunately, no simple generalisation of the result for the terms on the left-hand side twisted by the central binomial coefficients is known, even in the particular case $x=y$.

With the help of Clausen's identity

$$
P_{n}(y)^{2}={ }_{3} F_{2}\left(\begin{array}{c}
-n, n+1, \\
1,1
\end{array} \mid 1-y^{2}\right)=\sum_{k=0}^{n}\left(\begin{array}{l}
n \\
k
\end{array}\right)\left(\begin{array}{c}
n+k \\
n
\end{array}\right)\left(\begin{array}{c}
2 k \\
k
\end{array}\right)\left(-\frac{1-y^{2}}{4}\right)^{k},
$$

we find that the second generating function in (1.1) is equivalent to

$$
\sum_{n=0}^{\infty}\left(\begin{array}{c}
2 n \\
n
\end{array}\right) z^{n} \sum_{k=0}^{n}\left(\begin{array}{l}
n \\
k
\end{array}\right)\left(\begin{array}{c}
n+k \\
n
\end{array}\right)\left(\begin{array}{c}
2 k \\
k
\end{array}\right) x^{k}
$$

where $x=-\left(1-y^{2}\right) / 4$. In view of [14, Theorem 1], its Clausen-type specialisation [6] and our identities (1.5), (1.7) below, it is quite likely that the latter generating function can be written as a product of two arithmetic hypergeometric series, each satisfying a second-order linear differential equation. In this note we only recover the special case $z=x /(1+x)^{2}$ of the expected identity, the case which is suggested by [13, (VII1), (VII3)-(VII6)].

THEOREM 1.1. For $v$ in a small neighbourhood of the origin, take

$$
x(v)=\frac{v}{1+5 v+8 v^{2}} \quad \text { and } \quad z(v)=\frac{x(v)}{(1+x(v))^{2}}=\frac{v\left(1+5 v+8 v^{2}\right)}{(1+2 v)^{2}(1+4 v)^{2}} .
$$

Then

$$
\sum_{n=0}^{\infty}\left(\begin{array}{c}
2 n \\
n
\end{array}\right) z(v)^{n} \sum_{k=0}^{n}\left(\begin{array}{c}
n \\
k
\end{array}\right)\left(\begin{array}{c}
n+k \\
n
\end{array}\right)\left(\begin{array}{c}
2 k \\
k
\end{array}\right) x(v)^{k}=\frac{1+2 v}{1+4 v} \sum_{n=0}^{\infty} u_{n}\left(\frac{v}{(1+4 v)^{3}}\right)^{n},
$$


where the sequence [12, A183204]

$$
u_{n}=\sum_{k=0}^{n}\left(\begin{array}{l}
n \\
k
\end{array}\right)^{2}\left(\begin{array}{c}
n+k \\
n
\end{array}\right)\left(\begin{array}{c}
2 k \\
n
\end{array}\right)=\sum_{k=0}^{n}(-1)^{n-k}\left(\begin{array}{c}
3 n+1 \\
n-k
\end{array}\right)\left(\begin{array}{c}
n+k \\
n
\end{array}\right)^{3}
$$

satisfies the Apéry-like recurrence equation

$$
\begin{aligned}
& (n+1)^{3} u_{n+1}=(2 n+1)\left(13 n^{2}+13 n+4\right) u_{n}+3 n(3 n-1)(3 n+1) u_{n-1} \\
& \quad \text { for } n=0,1,2, \ldots, \quad u_{-1}=0, u_{0}=1 .
\end{aligned}
$$

Because $y^{2}=1+4 x$ for the second generating function in (1.1), the equivalent form of (1.5) is the identity

$$
\sum_{n=0}^{\infty}\left(\begin{array}{c}
2 n \\
n
\end{array}\right) P_{n}\left(\frac{\sqrt{(1+v)(1+8 v)}}{\sqrt{1+5 v+8 v^{2}}}\right)^{2}\left(\frac{v\left(1+5 v+8 v^{2}\right)}{(1+2 v)^{2}(1+4 v)^{2}}\right)^{n}=\frac{1+2 v}{1+4 v} \sum_{n=0}^{\infty} u_{n}\left(\frac{v}{(1+4 v)^{3}}\right)^{n} .
$$

We remark that Cooper constructs in [9, Theorem 3.1] a modular parametrisation of the generating function $\sum_{n=0}^{\infty} u_{n} w^{n}$. Namely, he proves that the substitution

$$
w(\tau)=\frac{\eta(\tau)^{4} \eta(7 \tau)^{4}}{\eta(\tau)^{8}+13 \eta(\tau)^{4} \eta(7 \tau)^{4}+49 \eta(7 \tau)^{8}}
$$

translates the function into the Eisenstein series $\left(7 E_{2}(7 \tau)-E_{2}(\tau)\right) / 6$. Here $\eta(\tau)=$ $q^{1 / 24} \prod_{m=1}^{\infty}\left(1-q^{m}\right)$ is Dedekind's eta function, $q=e^{2 \pi i \tau}$, and

$$
E_{2}(\tau)=\frac{12}{\pi i} \frac{d \log \eta}{d \tau}=1-24 \sum_{n=1}^{\infty} \frac{q^{n}}{1-q^{n}}
$$

Using this, Cooper derives a general family [9, Eqs. (37), (39)] of related Ramanujantype identities for $1 / \pi$. It is this result and the 'translation' method [15] which allow us to prove observations [13, (VII1), (VII3)-(VII6)]. Note that this modular parametrisation and results of Chan and Cooper [4, Lemmas 4.1 and 4.3] lead to the following hypergeometric forms of the generating function:

$$
\begin{aligned}
& \frac{1}{\sqrt{1+13 h+49 h^{2}}} \sum_{n=0}^{\infty} u_{n}\left(\frac{h}{1+13 h+49 h^{2}}\right)^{n} \\
& =\frac{1}{\sqrt{1+245 h+2401 h^{2}}} 3 F_{2}\left(\frac{1}{6}, \frac{1}{2}, \frac{5}{6} \mid \frac{1728 h}{\left(1+13 h+49 h^{2}\right)\left(1+245 h+2401 h^{2}\right)^{3}}\right) \\
& =\frac{1}{\sqrt{1+5 h+h^{2}}}{ }_{3} F_{2}\left(\frac{1}{6}, \frac{1}{2}, \frac{5}{6} \mid \frac{1728 h^{7}}{\left(1+13 h+49 h^{2}\right)\left(1+5 h+h^{2}\right)^{3}}\right)
\end{aligned}
$$

which are valid near the origin. 
Theorem 1.2 (Satellite identity). The identity

$$
\begin{aligned}
\sum_{n=0}^{\infty}\left(\begin{array}{c}
2 n \\
n
\end{array}\right)\left(\frac{x}{(1+x)^{2}}\right)^{n} \sum_{k=0}^{n}\left(\begin{array}{c}
n \\
k
\end{array}\right)\left(\begin{array}{c}
n+k \\
n
\end{array}\right)\left(\begin{array}{c}
2 k \\
k
\end{array}\right) x^{k} \\
\quad \times(2 x(3+4 x)-n(1-x)(3+5 x)+4 k(1+x)(1+4 x))=0
\end{aligned}
$$

is valid whenever the left-hand side makes sense.

\section{Proofs of Theorems 1.1 and 1.2}

The identity (1.5) is equivalent to

$$
\begin{aligned}
& \sum_{n=0}^{\infty}\left(\begin{array}{c}
2 n \\
n
\end{array}\right) \frac{v^{n}\left(1+5 v+8 v^{2}\right)^{n}}{(1+2 v)^{2 n+1}(1+4 v)^{2 n+1}} \sum_{k=0}^{n}\left(\begin{array}{l}
n \\
k
\end{array}\right)\left(\begin{array}{c}
n+k \\
n
\end{array}\right)\left(\begin{array}{c}
2 k \\
k
\end{array}\right) \frac{v^{k}}{\left(1+5 v+8 v^{2}\right)^{k}} \\
& \quad=\sum_{n=0}^{\infty} u_{n} \frac{v^{n}}{(1+4 v)^{3 n+2}} .
\end{aligned}
$$

It is routine to verify that both sides are annihilated by the differential operator

$$
\begin{gathered}
v^{2}(1+v)(1+8 v)\left(1+5 v+8 v^{2}\right) \frac{d^{3}}{d v^{3}}+3 v\left(1+21 v+122 v^{2}+280 v^{3}+192 v^{4}\right) \frac{d^{2}}{d v^{2}} \\
+\left(1+50 v+454 v^{2}+1408 v^{3}+1216 v^{4}\right) \frac{d}{d v}+4\left(1+22 v+108 v^{2}+128 v^{3}\right),
\end{gathered}
$$

and the proof of Theorem 1.1 follows. A similar routine shows the vanishing in Theorem 1.2.

\section{Sun's formulas}

In Table 1 we list the relevant parametrisations of Sun's formulas from [13]. The last column corresponds to the choice of $\tau$ in (1.6) such that $v /(1+4 v)^{3}=w(\tau)$ there. The general formulas for $1 / \pi$ in these cases,

$$
\sum_{n=0}^{\infty}(a+b n) u_{n} w^{n}=\frac{1}{\pi \sqrt{7}},
$$

are given by Cooper in [9, Eq. (37)]. On using (1.5) and its $v$-derivative

$$
\begin{aligned}
\sum_{n=0}^{\infty}\left(\begin{array}{c}
2 n \\
n
\end{array}\right) z(v)^{n} \sum_{k=0}^{n}\left(\begin{array}{l}
n \\
k
\end{array}\right)\left(\begin{array}{c}
n+k \\
n
\end{array}\right)\left(\begin{array}{c}
2 k \\
k
\end{array}\right) x(v)^{k} \\
\quad \times\left(n \frac{\left(1-8 v^{2}\right)\left(1+4 v+8 v^{2}\right)}{v(1+2 v)(1+4 v)\left(1+5 v+8 v^{2}\right)}+k \frac{1-8 v^{2}}{v\left(1+5 v+8 v^{2}\right)}\right) \\
=\frac{1+2 v}{1+4 v} \sum_{n=0}^{\infty} u_{n} \frac{v^{n}}{(1+4 v)^{3 n}}\left(n \frac{1-8 v}{v(1+4 v)}-\frac{2}{(1+2 v)(1+4 v)}\right),
\end{aligned}
$$


TABLE 1. The choice of parameters for observations in [13, p. 23]. The last column corresponds to the choice of $\tau$ such that $w(\tau)=v /(1+4 v)^{3}$ for the modular function $w(\tau)$ defined in (1.6).

\begin{tabular}{|c|c|c|c|c|c|}
\hline \# in [13] & $x$ & $z$ & $v$ & $w=v /(1+4 v)^{3}$ & $\tau$ \\
\hline (VII1) & $-\frac{1}{14}$ & $\frac{14}{225}$ & 1 & $\frac{1}{5^{3}}$ & $\frac{2 i}{\sqrt{7}}$ \\
(VII2) & $\frac{9}{20}$ & $-\frac{5}{196}$ & & & \\
(VII3) & $-\frac{1}{21}$ & $\frac{21}{484}$ & $1+\frac{\sqrt{14}}{4}$ & $\frac{188-42 \sqrt{14}}{22^{3}}$ & $\frac{i \sqrt{6}}{\sqrt{7}}$ \\
(VII4) & $-\frac{1}{45}$ & $\frac{45}{2116}$ & $\frac{5}{2}+\frac{7 \sqrt{2}}{4}$ & $\left(\frac{8-3 \sqrt{2}}{46}\right)^{3}$ & $\frac{i \sqrt{10}}{\sqrt{7}}$ \\
(VII5) & $\frac{1}{7}$ & $-\frac{7}{36}$ & $-\frac{3}{4}-\frac{\sqrt{7}}{4}$ & $\frac{-34+14 \sqrt{7}}{6^{3}}$ & $\frac{i \sqrt{3}}{\sqrt{7}}$ \\
(VII6) & $\frac{1}{175}$ & $-\frac{175}{30276}$ & $-\frac{45}{4}-\frac{17 \sqrt{7}}{4}$ & $\left(\frac{-13+7 \sqrt{7}}{174}\right)^{3}$ & $\frac{i \sqrt{19}}{\sqrt{7}}$ \\
(VII7) & $-\frac{576}{3025}$ & $\frac{3025}{188356}$ & & & \\
\hline
\end{tabular}

the equalities (3.1) together with the related specialisations of (1.7) (to eliminate the linear term in $k$ ) imply Sun's identities (VII1), (VII3)-(VII6) by translation [15].

Note that Cooper's [9, Table 1] involves two more examples corresponding to the choices $-1 / 4^{3}$ and $-1 / 22^{3}$ for $v /(1+4 v)^{3}$; the values of $x$ and $z$ in these cases are zeros of certain irreducible cubic polynomials though. There are also several examples when $x$ and $z$ are taken from a quadratic field. For instance, taking $\tau=i \sqrt{11} / \sqrt{7}$, one gets

$$
x=\frac{23-8 \sqrt{11}}{175} \quad \text { and } \quad z=\frac{83-32 \sqrt{11}}{1100}
$$

in (1.5) and (1.7); the corresponding $v_{1}=-6.798 \ldots$ and $v_{2}=-0.018 \ldots$ solve the quartic equation $64 v^{4}+448 v^{3}+96 v^{2}+56 v+1=0$. As such identities are only of theoretical importance, we do not derive them here.

\section{Generating functions of other special polynomials}

It is apparent that there is a variety of formulas similar to (1.5) and (1.7) designed for generating functions of other polynomials. For example, Sun's list contains five identities involving values of the polynomials

$$
A_{n}(x)=\sum_{k=0}^{n}\left(\begin{array}{l}
n \\
k
\end{array}\right)^{2}\left(\begin{array}{c}
n+k \\
n
\end{array}\right) x^{k}, \quad n=0,1,2, \ldots
$$

By examining the entries (2.1)-(2.3) on [13, p. 3] one notices that the parameters $x$ and $z$ of the generating function

$$
\sum_{n=0}^{\infty}\left(\begin{array}{c}
2 n \\
n
\end{array}\right) A_{n}(x) z^{n}
$$


are related by $z=x /(1-4 x)$, while the entries $(6.1)$ and (6.2) on [13, p. 15] there correspond to the relation $z=1 /(x+1)^{2}$. With some work we find that those specialisations indeed lead to third-order arithmetic linear differential equations which can be then identified with the known examples [5, 8]:

$$
\begin{aligned}
& \sum_{n=0}^{\infty}\left(\begin{array}{c}
2 n \\
n
\end{array}\right) \frac{v^{n}(1-v)^{n}(1-4 v)^{n}}{\left(1-2 v+4 v^{2}\right)^{2 n+1}} \sum_{k=0}^{n}\left(\begin{array}{c}
n \\
k
\end{array}\right)^{2}\left(\begin{array}{c}
n+k \\
n
\end{array}\right) \frac{v^{k}(1-v)^{k}(1-4 v)^{k}}{\left(1-4 v^{2}\right)^{2 k+1}} \\
& \quad=\sum_{n=0}^{\infty} \sum_{k=0}^{n}\left(\begin{array}{l}
n \\
k
\end{array}\right)^{2}\left(\begin{array}{c}
n+k \\
n
\end{array}\right)^{2} \frac{v^{n}(1-2 v)^{n}(1-4 v)^{2 n}}{(1-v)^{n+1}(1+2 v)^{n+1}} \\
& =\sum_{n=0}^{\infty} \sum_{k=0}^{n}\left(\begin{array}{l}
n \\
k
\end{array}\right)^{2}\left(\begin{array}{c}
2 k \\
k
\end{array}\right)\left(\begin{array}{c}
2 n-2 k \\
n-k
\end{array}\right) \frac{(-1)^{n} v^{n}(1-v)^{n}\left(1-4 v^{2}\right)^{n}}{(1-4 v)^{2 n+2}} \\
& =\sum_{n=0}^{\infty} \frac{(3 n) !}{n !^{3}}\left(\begin{array}{c}
2 n \\
n
\end{array}\right) \frac{v^{n}(1-v)^{n}\left(1-4 v^{2}\right)^{n}(1-4 v)^{4 n}}{\left(1+4 v-8 v^{2}\right)^{2 n+2}}
\end{aligned}
$$

and

$$
\begin{gathered}
\sum_{n=0}^{\infty}\left(\begin{array}{c}
2 n \\
n
\end{array}\right) \frac{v^{2 n}}{\left(1+10 v+27 v^{2}\right)^{2 n+1}} \sum_{k=0}^{n}\left(\begin{array}{l}
n \\
k
\end{array}\right)^{2}\left(\begin{array}{c}
n+k \\
n
\end{array}\right) \frac{\left(1+9 v+27 v^{2}\right)^{k}}{v^{k}} \\
=\sum_{n=0}^{\infty} \frac{(3 n) !}{n !^{3}}\left(\begin{array}{c}
2 n \\
n
\end{array}\right) \frac{v^{n}\left(1+9 v+27 v^{2}\right)^{n}}{(1+9 v)^{6 n+2}},
\end{gathered}
$$

respectively. Additionally, there are satellite identities for each of the specialisations, both similar to (1.7). These identities, the known Ramanujan-type formulas for the right-hand sides and the translation technique can be then used to prove Sun's observations.

On the other hand, as already mentioned at the beginning, it is natural to expect the existence of Bailey-Brafman-like identities [7, 14] for the two-variate generating functions (1.4), (4.1).

\section{A question}

Given an (arithmetic) generating function $\sum_{n=0}^{\infty} A_{n} z^{n}$ which satisfies a second-order linear differential equation (with regular singularities), is it true that $\sum_{n=0}^{\infty}\left(\begin{array}{c}2 n \\ n\end{array}\right) A_{n} z^{n}$ can be written as the product of two arithmetic series, each satisfying (its own) secondorder linear differential equation?

Here, of course, we allow $A_{n}$ to depend on some other parameters; the example of such a product decomposition for $A_{n}=A_{n}(x)=\sum_{k}\left(\begin{array}{l}n \\ k\end{array}\right)^{2}\left(\begin{array}{c}2 k \\ n\end{array}\right) x^{k}$ has been given recently by Rogers and Straub [11, Theorem 2.3]. An affirmative answer to the question would give one an arithmetic parametrisation of the generating function $\sum_{n=0}^{\infty}\left(\begin{array}{c}2 n \\ n\end{array}\right) P_{n}(x) P_{n}(y) z^{n}$ (see (1.2) or (1.3)). 
Note that there are some other generating functions in [13], like the first one in (1.1), which are not of the form $\sum_{n=0}^{\infty}\left(\begin{array}{c}2 n \\ n\end{array}\right) A_{n} z^{n}$. We believe, however, that they can be reduced to the latter form by a suitable algebraic transformation.

\section{Acknowledgements}

I would like to thank Heng Huat Chan, Jesús Guillera and James Wan for our fruitful conversations on the subject, and Shaun Cooper for useful comments and for making me familiar with his work [9] while this paper was in preparation. I express my gratitude to the anonymous referee for several valuable comments which helped me to improve the presentation.

\section{References}

[1] W. N. Bailey, Generalized Hypergeometric Series, Cambridge Mathematical Tracts 32 (Cambridge University Press, Cambridge, 1935).

[2] W. N. Bailey, 'The generating function of Jacobi polynomials', J. Lond. Math. Soc. 13 (1938), 8-12.

[3] F. Brafman, 'Generating functions of Jacobi and related polynomials', Proc. Amer. Math. Soc. 2 (1951), 942-949.

[4] H. H. Chan and S. Cooper, 'Eisenstein series and theta functions to the septic base', J. Number Theory 128 (2008), 680-699.

[5] H. H. Chan and S. Cooper, 'Rational analogues of Ramanujan's series for $1 / \pi$ ', Math. Proc. Cambridge Philos. Soc. 153 (2012), 361-383.

[6] H. H. Chan, Y. Tanigawa, Y. Yang and W. Zudilin, 'New analogues of Clausen's identities arising from the theory of modular forms', Adv. Math. 228 (2011), 1294-1314.

[7] H. H. Chan, J. Wan and W. Zudilin, 'Legendre polynomials and Ramanujan-type series for $1 / \pi$ ', Israel J. Math. (2013), to appear, doi:10.1007/s11856-012-0081-5.

[8] H. H. Chan and W. Zudilin, 'New representations for Apéry-like sequences', Mathematika 56 (2010), 107-117.

[9] S. Cooper, 'Sporadic sequences, modular forms and new series for $1 / \pi$ ', Ramanujan J. 29 (2012), $163-183$

[10] L. C. Maximon, 'A generating function for the product of two Legendre polynomials', Norske Vid. Selsk. Forh. Trondheim 29 (1956), 82-86.

[11] M. Rogers and A. Straub, 'A solution of Sun's \$520 challenge concerning 520/ $\pi$ ', Preprint, arXiv:1210.2373, 2012.

[12] N. J. A. Sloane, The On-Line Encyclopedia of Integer Sequences, published electronically at http: //www.research.att.com/ njas/sequences/, 2012.

[13] Z.-W. Sun, 'List of conjectural series for powers of $\pi$ and other constants', Preprint, arXiv:1102.5649v37, 24 January 2012.

[14] J. Wan and W. Zudilin, 'Generating functions of Legendre polynomials: a tribute to Fred Brafman', J. Approx. Theory 164 (2012), 488-503.

[15] W. Zudilin, 'Lost in translation', Proceedings of the Waterloo Workshop in Computer Algebra (W80) (May 2011), In Honour of Herbert S. Wilf, Springer Proceedings in Mathematics and Statistics (eds. I. Kotsireas and E. V. Zima) (Springer, New York, 2013), to appear.

WADIM ZUDILIN, School of Mathematical and Physical Sciences, The University of Newcastle, Callaghan, NSW 2308, Australia e-mail: wadim.zudilin@newcastle.edu.au 